\title{
EVENT DRIVEN RECOMMENDATION SYSTEM FOR E-COMMERCE USING KNOWLEDGE BASED COLLABORATIVE FILTERING TECHNIQUE
}

\author{
MAHESH KUMAR SINGH*AND OM PRAKASH RISHI ${ }^{\dagger}$
}

\begin{abstract}
The Internet is changing the method of selling and purchasing items. Nowadays online trading replaces offline trading. The items offered by the online system can influence the nature of buying customers. The recommendation system is one of the basic tools to provide such an environment. Several techniques are used to design and implement the recommendation system. Every recommendation system passes from two phases similarity computation among the users or items and correlation between target user and items. Collaborative filtering is a common technique used for designing such a system. The proposed system uses a knowledge base generated from knowledge graph to identify the domain knowledge of users, items, and relationships among these, knowledge graph is a labelled multidimensional directed graph that represents the relationship among the users and the items. Almost every existing recommendation system is based on one of feature, review, rating, and popularity of the items in which users' involvement is very less or none. The proposed approach uses about 100 percent of users' participation in the form of activities during navigation of the web site. Thus, the system expects under the users' interest that is beneficial for both seller and buyer. The proposed system relates the category of items, not just specific items that may be interested in the users. We see the effectiveness of this approach in comparison with baseline methods in the area of recommendation system using three parameters precision, recall, and NDCG through online and offline evaluation studies with user data, and its performance is better than all other baseline systems in all aspects.
\end{abstract}

Key words: Recommendation System (RS), Collaborative Filtering (CF), Knowledgebase, Knowledge graph. e-Commerce, Subject predicate object (SPO), K- Nearest Neighborhood (KNN), Normalized Discounted Cumulative Gain (NDCG), Bayesian Personalized Ranking(BPR), Visual Bayesian Personalized Ranking (VBPR), Deep Convolutional Neural Network (DCNN), Joint Representation Learning (JRL).

AMS subject classifications. 68M11

1. Introduction. This is the era of I-way. With the development of high-speed Internet, smart computing technology, and artificial intelligence, e-Commerce is growing very rapidly. It tries to replace traditional selling and purchasing methods. It provides more choices to the user for purchasing items in easy and efficient ways. Many e-Commerce companies are available through the world like Amazon, Tokopedia, Flipcart, Bukalap, Netflix, Snapdeal, and so on are using the recommendation system to help their users. The choices provided by these e-Commerce companies influence the user's decision in buying the products. Since the number of users increases exponentially, hence it is the challenge of e-Commerce companies to handle those. Due to multiples choices of similar types of products user generally confused and irritated, to overcome these problems, companies are trying to develop or upgrade a system to guide the users in very efficient ways. The recommendation system is a technique by which the system suggests the relevant products to the customers in reputed Ecommerce [1] companies, where there is a large verity of products are available. The recommendation system can be personalized or non-personalized. The non-personalized system commonly used in a physical store that processed the common features of the products like the popularity of the product for example actors in a movie. On the other hand, the recommendation system that uses the navigation details and the activities in the form of rating/review of the products given by the customers is called a personalized [2] recommendation system. The product can be recommended to a customer if the calculated preference score is high. If it is to consider that each product or service has a different page then page rank provides the rating of the similar product of different brand names by using page ranking concept, we can calculate the popularity of the product or service. Nowadays every E-commerce site wants to use the recommendation system that is used to serve the millions

* Department of Computer Science and Informatics. University of Kota, Rajasthan, India. (maheshkrsg@gmail.com).

$\dagger$ Department of Computer Science and Informatics. University of Kota, Rajasthan, India. (omprakashrishi@yahoo.com). 
TABLE 1.1

List of some popular websites that are using recommendation system to facilitate their users.

\begin{tabular}{|c|c|l|l|l|}
\hline Sr. No. & Name of site & User & Item & Description \\
\hline 1 & Linkedin & Member & Members or Jobs & Members are interested in other members or jobs \\
2 & Facebook & Member & Members & Members are interested in another member \\
3 & Amazon & Member & Products or books & Members are interested in products \\
4 & Netflix & Member & Movies or Story series & Members are interested in watching movies or story series. \\
5 & Flipcart & Member & Products & Members are interested in products. \\
\hline
\end{tabular}

of customers. It increases the customers' faithfulness and directs them to new items in the product catalog. Very popular E-commerce site Amazon attracts large community using user-centric recommendation system by accessing the user history data, while small scale company faces the problems extensive shopping history record since many customers are one-time visitors. Some popular sites where the recommendation is working based on user and item as shown in Table 1.1.

Recommendation system [3] processes user's information stored in the system during navigation of the e-Commerce site and provide the most relevant product to the user and solve his/her problems. Let $\mathrm{U}$ be the set of users and I be the set of items, then prediction function $\mathrm{P}$ is defined as $P: U X I->R$ where $\mathrm{R}$ be the list of items for a specific user. There are so many techniques that are used in designing recommendation systems, including popularity, based, content-based filtering [4], collaborative filtering [5], graph-based filtering [6], and demographic filtering [7]. In this study, we use a knowledge-based recommendation collaborative filtering technique that processes the personalized heterogeneous information in the form of triplets ( $u, r, p)$ that are generated when user $\mathrm{u}$ interact with the product $\mathrm{p}$ with $\mathrm{r}$ activity, stored in the system.

Whole paper is divided into four sections. The first section contains a literature survey that discusses the latest research paper in this area, motivation, and objective of this paper. Second section deals model or method which is used in this research work. The third section is the result and discussion section that deals with model implementation and comparison with baseline systems. The fourth section includes the conclusion and future scope of the paper.

2. Related Work. The recommendation system tries to process the historical data of users or features of items and provides the best relevant result for a particular user. It is used in almost various fields to help users to decide like a restaurant recommendation system provides certain preferences [8] of restaurants, book recommendations [9] provides a list of most preferred books, social network [10] most relevant friends, movie recommendation [11] and widely used product recommendation [12] in e-commerce recommendation system to recommend the item to the user based on ranking. The recommendation for publication [13, 14] that help authors decide where they should submit their manuscripts in Content-based Journals and Conferences. This system recommends suitable journals or conferences with a priority order based on the abstract of a manuscript. Proposed a three-step hybrid ranking [15] order system for finding the top-N list for the multi-criteria recommendation system this approach is good for both the traditional no-ranking item-based collaborative recommendation and single-criteria-ranking approach that uses two popular learning-to-rank methods. Recommender System on computer science. The recommendation system techniques are mainly classified [16] into two categories personalized and non-personalized. Since a recommendation system is created for any customer for his/her likes and dislikes, hence non-personalized recommendation system is negligible, there is no or very limited scope for the researchers. Personalized recommendation systems [17] are used user's profile that is userbased and provides lists of different items while non- personalized recommendation system uses the popularity of the items and provides a similar list for all users.

Several techniques are used in the development and design of a recommendation system which includes, collaborative filtering, content-based filtering, and hybrid filtering technique (see fig 2.1). Collaborative filtering is a widely used tool in the development of a recommendation system based on user's action and profiling to generate similarities in data, there are two types of collaborative filters, memory-based and model-based. The similarity in collaborative filtering is computed in two different ways user-user similarity and item-item similarity using classifiers [18] and clustering algorithms. Content-based filtering [19] technique used features of items to find similarity, hybrid filtering technique uses both features content and collaborative. Similarity descriptions 


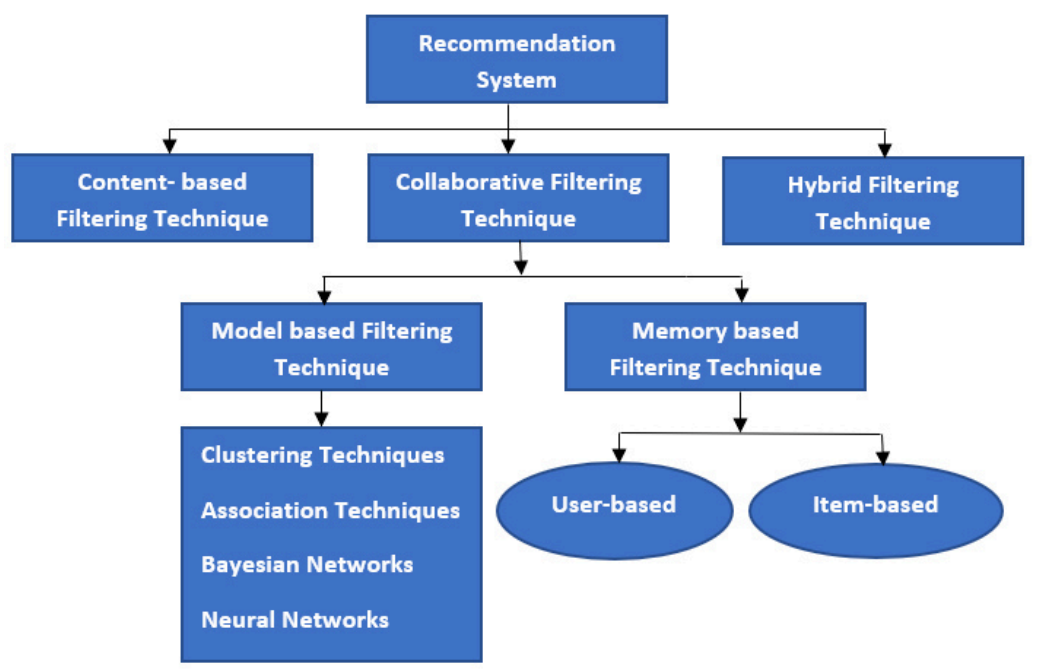

FIG. 2.1. Classification of Recommendation Systems

and factors can influence the decision of product purchasing. Most of the recommendation system techniques are used homogeneous data in the form of a review or rating $[18,20]$ given by the users to the items, but there are very few users that are ready to rate or review the items while they are bought and used the items. Hence, a recommendation system based on review and ratings [18] is not producing an accurate list of items as he/she required. Image-based recommendation [21] used the appearance of the items to find the similarity. One more problem of the recommendation system is that product is bought or returned by the user.

2.1. Motivation. Artificial intelligence, machine learning, and deep learning are the current growing technologies. There are so many opportunities in these areas for the researchers. E-commerce is rapidly growing technology nowadays due to the availability of high-performance computing devices, and efficient systems. Information overload is one of the main problems for both enterprise as well as users, but recommendation system (RS) play a vital role to minimize it. Almost every E-Commerce company is using it, so efficient recommendation system design and development is one of the key features for researchers nowadays. There are two approaches used to design and implementation of RS, classical programming approach, and machine learning approach. The classical approach is used for small data set while the machine learning approach is used in a large data set. Here we use the classical programming approach. There are so many RS based on popularity, rating, and review but, we know that only 20 to 30 percent of users are participating in review and rating.

The proposed system uses maximum user involvement by the navigation of web site in the form of product view, selection, and purchasing, these heterogeneous data play a vital role in creating a recommendation system.

3. Methodology. The methodology used in the proposed system is shown in figure 3.1. Data is the backbone of any predictive system. The primary aim of any system is the collection of quality data. Data can be collected in two ways, implicit and explicit. Explicit are those data which are provided by the user required by e-Commerce web sites in the form of registration, reviews comments, and rating, etc. Implicit data can be stored by e-Commerce sites automatically using cookies or any other methods implemented by the system, that contains the user's navigation details and activities performed by the user during surfing the e-Commerce website. Knowledge base [22] is a type of implicit data that is collected by the e-Commerce website. Every web site is a collection of web pages connected and formed liked a directed graph, each page is reserve for each product that shows the description of that product, types of links describe the types of activities. Domain knowledge monitors the activities performed by users like purchase transactions, clicks, etc.

The knowledge base is created with the help of a knowledge graph which is, a directed graph that shows relationships among items-users and items-items. In the given data set, set of items and set of users denoted 


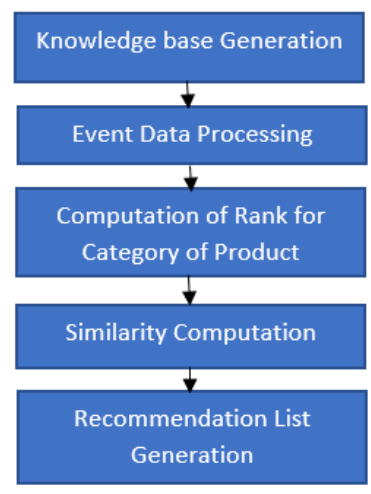

FIG. 3.1. Methodology

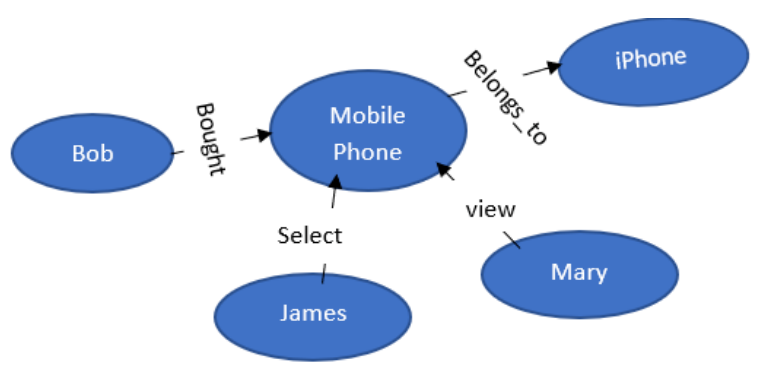

FIG. 3.2. Knowledge Graph

by vertices and set of relationships by edges.

Suppose there is a sequence of events as Bob bought a mobile phone of the brand iPhone, James selects the same mobile and Mary only views that mobile phone then this information can be represented by a knowledge graph. The facts of the knowledge base for these events are written as Buys(Bob, Mobile_Phone), belongs_to(Mobile_Phone, iPhone), Selects(James, Mobile_Phone), Views(Mary, Mobile_Phone), as shown in figure 3.2 .

Event Data Preprocessing is a module that processes the knowledge base as per the requirement of the system. The event attribute contains three types of entries view, select, and buy. Transform data as separate columns as buy, select and view as the tuple (buy, select, view), if any user buys then it is considered as $(1,1,1)$, select $(0,1,1)$, and view as $(0,0,1)$ respectively. A user i can interact with item $\mathrm{j}$ in many ways like view, select, buy, also_buy, etc., only three events are considered for the computation of user interaction score or preference rank of the item. The weight of events as buy $>$ select $>$ view is considered.

The calculation of preference score or rank of each class of items is formulated with the equation (3.1):

$$
C_{u p}=X \frac{C_{u p}^{v}}{\sqrt{\sum_{p=1}^{n}\left(C_{u p}^{v}\right)^{2}}}+Y \frac{C_{u p}^{s}}{\sqrt{\sum_{p=1}^{n}\left(C_{u p}^{s}\right)^{2}}}+Z \frac{C_{u p}^{b}}{\sqrt{\sum_{p=1}^{n}\left(C_{u p}^{b}\right)^{2}}}
$$

where $C_{u p}^{v}$ denotes user $u$ viewed item/product $p, C_{u p}^{s}$ user $u$ select item/product $p$ and $C_{u p}^{b}$ user $u$ bought item/product $p, X, Y$ and $Z$ are weight adjusting constants, for better result $X=0.25, Y=0.5$ and $Z=1.0$.

\section{Algorithm:}

Input: Entity set E (Set of products $\mathrm{P}$ and Set of users U), Relation Set R, triplet set (u,r,p) is in S (ordered set).

Output: List top $\mathrm{N}$ products recommendation to the corresponding user as per user's information. Begin 
Step1: Let $\mathrm{N}$ is the number product classes and $\mathrm{M}$ are the number of users compute preference score of each product class by formula (3.1).

Step2: Calculate similarity of the users by the formula (4.1).

Step3: Calculate prediction score $\operatorname{Pre}(\mathrm{u}, \mathrm{p})$ of product $\mathrm{p}$ with respect to user u using formula (4.2).

Step4: Select top N class of products based for user $\mathrm{u}$ on the basis of value of Pre $(\mathrm{u}, \mathrm{p})$ with preference score .

Step4: If any product is already bought by the user then replace it with other product and form the list of recommendation.

Step5: Prepare most recommended products for enterprise. .

End

4. Experiments and Results. In our study, we develop our method working with retail e-Shop that sells mobile phones and home appliances only electronics items, about 200 users visit daily but 50 percent of them only visit or view the items, hence user-item bought or select data are very sparse that are ignored. Only 50 percentage data are considered in which at least one event must be bought or select that show their interest. Consider a data set that stores events of users, contains 20680 events (i.e. Buy, select, view) performed by 1240 users with unique user_IDs on 114 class products with unique product_IDs with the multiple numbers of products, of two categories (i.e. mobile phones and electronics items) and brands (i.e. Nokia, iPhone, Sony, Vivo and RedMe, Tata). The event data set contains five attributes User_ID, event, product_ID, Brand, and Category as shown in table 4.1. We use Jupiter notebook for providing coding environment,python 3.0 for programming, pandas [23] python library for data wrangling or cleansing and analysis, matplotlib for visualization, NumPy for numerical computation, sci-kit-learn, scipy.sparse for sparse matrices. We use a memory-based collaborative filtering technique.

4.1. Wrangling or Cleansing of Data. In the selected data set event attribute contains different types of activities performed by users during navigation of web site. We have to categorize these events into three main categories buy, select and view, because they have different weights in preference computation, and binary formation of these entries in the data set. After expansion and transformation result shown in table 4.2.

4.2. Computation of Preference Rank. Computation of preference rank of each class of products, sub-setting of data-frame applied on product_ID. The preference rank of the product depends on the number of users interact with the product. It is computed by using equation (3.1). This shows the performance of the product. Product with product_ID 11278 is the highest performing and product_ID 33000 is least performing products as shown in table 4.3.

4.3. Similarity Computation. Similarity computation can be done in two ways, user-user similarity and item-item similarity. The similarity between target user $\mathrm{u}$ and a neighbor $\mathrm{v}$ can be computed using Pearson's Correlation coefficient [24] as equation (4.1). Ignoring all least performing items that are less than 0.75 preference score.

$$
\operatorname{sim}(u, v)=\frac{\sum_{p \varepsilon P}\left(r_{u, p}-\overline{r_{u}}\right) X\left(r_{v, p}-\overline{r_{v}}\right)}{\sqrt{\sum_{i \varepsilon I}\left(r_{u, i}-\overline{r_{u}}\right)^{2}} X \sqrt{\sum_{p \varepsilon P}\left(r_{v, p}-\overline{r_{v}}\right)^{2}}}
$$

TABLE 4.1

Event table

\begin{tabular}{|c|c|c|c|c|}
\hline UserID & Event & ProductID & Brand & Category \\
\hline 14893 & Buy & 11277 & sony & Electronics_item \\
48958 & view & 11378 & RedMe & Mobile \\
48357 & Buy & 11278 & iPhone & Mobile \\
55674 & select & 11378 & RedMe & Mobile \\
48357 & View & 21277 & Sony & Electronics_item \\
48360 & View & 11278 & iPhone & Mobile \\
48730 & select & 21277 & Sony & Electronics_item \\
48351 & View & 11278 & iPhone & Mobile \\
. &. &. &. &. \\
. &. &. &. &. \\
\hline
\end{tabular}


TABLE 4.2

After Expansion and Binary formation table in preprocessing

\begin{tabular}{|c|c|c|c|c|c|c|}
\hline UserID & Buy & Select & View & ProductID & Brand & Category \\
\hline 14893 & 1 & 1 & 1 & 11277 & sony & Electronics_item \\
48958 & 0 & 0 & 1 & 11378 & RedMe & Mobile \\
48357 & 1 & 1 & 1 & 11278 & iPhone & Mobile \\
55674 & 0 & 1 & 1 & 11378 & RedMe & Mobile \\
48357 & 0 & 0 & 1 & 21277 & Sony & Electronics_item \\
48360 & 0 & 0 & 1 & 11278 & iPhone & Mobile \\
48730 & 0 & 1 & 1 & 21277 & Sony & Electronics_item \\
48351 & 0 & 0 & 1 & 11278 & iPhone & Mobile \\
4853 & 1 & 1 & 1 & 21309 & Tata & Electronics_item \\
464056 & 0 & 1 & 1 & 21303 & Nokia & mobile \\
483575 & 0 & 0 & 1 & 21301 & Nokia & mobile \\
$\cdot$ & $\cdot$ & $\cdot$ & $\cdot$ & $\cdot$ & $\cdot$ & . \\
. & $\cdot$ &. & $\cdot$ &. & \multicolumn{2}{c}{} \\
\hline
\end{tabular}

TABLE 4.3

Product with its Preference Rank

\begin{tabular}{|c|c|c|c|}
\hline ProductID & Brand & Category & Preference Rank \\
\hline 11278 & iPhone & Mobile & 6.1013572 \\
11277 & sony & Electronics_item & 5.4464 \\
44575 & iPhone & Mobile & 2.41421356 \\
34275 & Nokia & Mobile & 2.25829 \\
33488 & RedMe & Mobile & 3.07565139 \\
12453 & VIVO & Mobile & 3.30039626 \\
14575 & iPhone & Mobile & 3.0913 \\
11378 & RedMe & Mobile & 6.0655 \\
21266 & Sony & Electronics_item & 3.6670 \\
21277 & Sony & Electronics_item & 5.49658159 \\
22458 & VIVO & Mobile & 3.83711731 \\
24555 & iPhone & Mobile & 1.75 \\
33000 & RedMe & Mobile & 0.35355339 \\
33277 & Sony & Electronics_item & 2.91421356 \\
33479 & RedMe & Mobile & 1.75 \\
21254 & sony & Electronics_item & 1.6771 \\
11279 & iPhone & Mobile & 1.75 \\
21278 & sony & Electronics_item & 5.7760 \\
21280 & sony & Electronics_item & 4.65690 \\
21284 & sony & Electronics_item & 5.6672 \\
21286 & sony & Electronics_item & 4.9983 \\
21309 & Tata & Electronics_item & 2.76862 \\
$\cdot$ & $\cdot$ & . &. \\
. &. & . &. \\
& & & \\
\hline
\end{tabular}

where $r_{u, p}$ and $r_{v, p}$ be rank given by user $u$ and $v$ to item $p, \overline{r_{u}}$ and $\overline{r_{v}}$ be the average rank of user $u$ and $v$. It is best suited for less number of users but the main problem with the user based is scalability when the number of users is very large then it very difficult to find the similarity among the users.

4.4. Recommendation List Generation. Using the equation (4.1), we can compute the ranking prediction of item or product $i$ for the target user $u$. Association of products with user play a vital role in recommendation list generation for the users. User-user based collaborative filtering (equation 4.2) and top $N$ recommendation technique [12] is used to generate the list of recommendation of items the user. Recommendation lists of each user are shown in table 4.4 .

$$
\operatorname{Pre}(u, p)=\overline{r_{u}}+\frac{\sum_{v \varepsilon V} \operatorname{sim}(u, v) X\left(r_{v, p}-\overline{r_{v}}\right)}{\sum_{v \varepsilon V}|\operatorname{sim}(u, v)|}
$$

where $\operatorname{Pre}(u, p)$ is the prediction score of item/product $p$ with user $\mathrm{u}, V$ is the set of all users that are similar to $u, r_{v, p}$ is ranking of user $v$ given to the item $p$. After computing similarity between items, we select as $k$ 
TABLE 4.4

List of Products Recommended to User

\begin{tabular}{|c|c|c|c|c|}
\hline UserID & ProductID & Brand & Category & Preference Rank \\
\hline 4830 & 11278 & iPhone & Mobile & 6.101357 \\
\hline . & 21277 & Sony & Electronics_item & 5.496582 \\
\hline . & 12453 & VIVO & Mobile & 3.300396 \\
\hline . & 33277 & Sony & Electronics_item & 2.914214 \\
\hline . & 34275 & Nokia & Mobile & 2.25829 \\
\hline . & 21254 & Sony & Electronics_item & 1.866025 \\
\hline 4890 & 21277 & sony & Electronics_item & 5.22129 \\
\hline . & 21266 & Tata & Electronics_item & 3.6670 \\
\hline . & 14575 & iPhone & Mobile & 3.0913 \\
\hline . & 33523 & iPhone & Mobile & 2.9352 \\
\hline 4852 & 11278 & iPhone & Mobile & 6.101357 \\
\hline . & 36000 & RedMe & Mobile & 2.914214 \\
\hline . & 44575 & iPhone & Mobile & 2.414214 \\
\hline 4893 & 11278 & iPhone & Mobile & 6.101357 \\
\hline$\cdot$ & 21277 & Sony & Electronics_item & 5.496582 \\
\hline . & 12453 & VIVO & Mobile & 3.300396 \\
\hline . & 33277 & Sony & Electronics_item & 2.914214 \\
\hline . & 34275 & Nokia & Mobile & 2.25829 \\
\hline$\cdot$ & 21254 & Sony & Electronics_item & 1.866025 \\
\hline . & · & · & · & . \\
\hline . & . & . & . & . \\
\hline
\end{tabular}

most similar items to the target item and generate prediction value of user $u$. Some users' recommendation lists are shown in table 4.4. The recommendation list for a user contains those products which are not bought by the user. High performing products always on the top of the list.

4.5. Evaluation Matrices. To evaluate the quality of the proposed system we assess the general performance using three most commonly used evaluation matrices [25] in the retrieval system, precision (Eq 4.3), recall (Eq 4.4), and Normalized Discounted Cumulative Gain (NDCG) (Eq 4.6). The recommendation is viewed as an information retrieval task i.e. retrieve all products which are predicted to be "good".

Precision is a measure of exactness, determines the fraction of relevant products retrieved out of all products retrieved.

$$
\text { Precision }=\frac{\mid \text { Good products recommended } \mid}{\mid \text { Total number of recommended products } \mid}
$$

It measures the system's ability to reject any non-relevant products in the retrieved set

Recall is a measure of completeness, determines the fraction of relevant products retrieved out of all relevant products.

$$
\text { Recall }=\frac{\mid \text { Good products recommended } \mid}{\mid \text { Total good products } \mid}
$$

It measures the system's ability to find all the relevant products in the retrieved set.

Discounted Cumulative Gain (DCG) is measured the usefulness or gain of a product based on its position in the result list. The DCG accumulated at a particular rank position $\mathrm{p}$ of any product.

$$
D C G_{p}=\sum_{i=1}^{p} \frac{2^{\text {rel }_{i}}-1}{\log _{2}(i+1)}
$$

Normalized Discounted Cumulative Gain (NDCG): performance from one query to next can not be consistently achieved using DCG only hence NDCG is used. It is done by sorting all relevant products by their relative relevance producing the maximum possible DCG through position $p$ of any product. NDCG is computed as

$$
N D C G_{p}=\frac{D C G_{p}}{I D C G_{p}}
$$




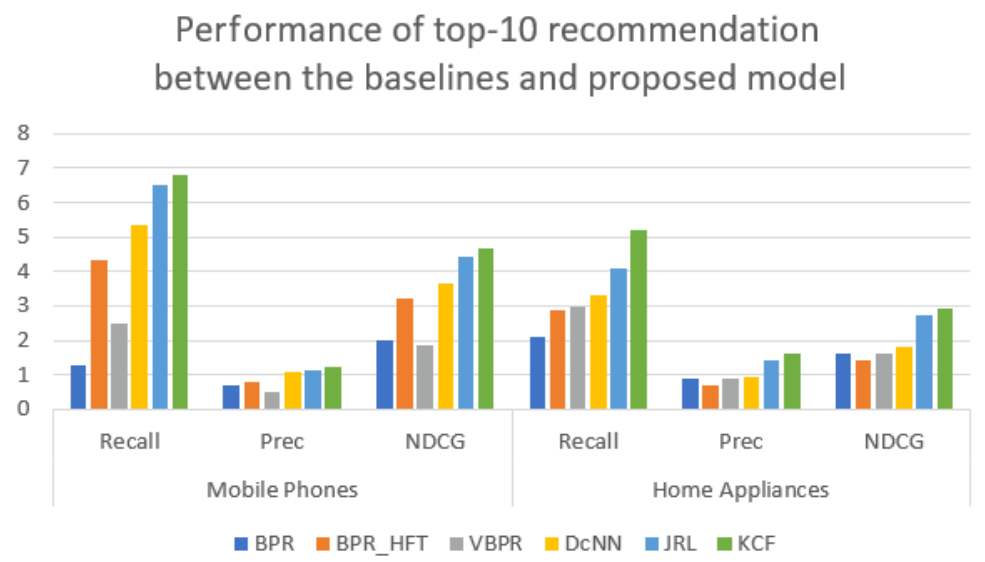

FIG. 4.1. Performance of top-10 recommendation between the baselines and Knowledge based collaborative filtering $(K C F)$

4.6. Comparisons with Baseline Methods. We choose commonly used representative and state-ofthe-art methods as the baselines for the performance comparisons.

BPR: The Bayesian Personalized Ranking [26] is a popular model used in top-N recommendation system, it is based on matrix factorization. It gives good results small data-set.

BPR_HFT: Hidden Factors and Topics (BPR_HFT) [27] is a method used for textual reviews; we use HFT under BPR pair wise ranking framework for fair comparison.

VBPR: Visual Bayesian Personalized Ranking ( VBPR) [28] is used for recommendation with images.

DeepCoNN: Deep Convolution Neural Network (DCNN) [29] is a review based deep recommendation method to jointly model the users and the products.

JRL: Joint Representation Learning (JRL) [30] is a model which can leverage multi-model information for Top- $\mathrm{N}$ recommendation.

The performance of the proposed system as well as the baseline models are shown in the performance graph (see fig. 4.1). The baseline models are categorized based on the input source of information which is review based (HFT and DeepCoNN), rating based (BPR), Image-based (VBPR), and heterogeneous information modeling JRL. The information is modeled as rating though buy relation, review though select relation and knowledge about the product through view, also view, belong to category, belong to brand relations.

From the experimental result, it is clear that both review based and rating based models enhanced the performance of recommendation system as compare to popularity based, but heterogeneous information sourcebased model like JRL performs much better than baseline systems, which gives the idea about the heterogeneity. Knowledge-based collaborative filtering (KCF) performs much better than that of JRL consistently overall evaluation measures that verify the proposed system. This improvement in the performance, due to more information sources, and better-structured knowledge graph to model the heterogeneous information. Figure 4.1 shows the comparison of KCF with the other baselines models from the comparison graph it is clear that KCF performs better as the selected baseline system models in the area of recommendation system.

Conclusion and Future Scope. Choices made by the e-Commerce web site must be considered, it influences the decision of the customer to buy the products. Hence it is the main focus of the recommendation system. The experimental results on the e-Shop simulated dataset show the promising performance of our proposed approaches in terms of the accuracy of the recommendation in comparison with the traditional baseline approaches. The proposed system focuses on users' event-driven approach, hence the participation of user increases as compare to those methods which are based on reviews or ratings because about 20 to 40 percent of users participated in review and rating while others are not interested but they still interact with the products. Therefore, the proposed system generates a more closed list as a recommendation as compared to other such systems. This research very useful for small scale enterprises like retailers since it requires storing only user navigation details to generate the ranking score of the item, and item details to recommend the list of items in 
the future. For future work, several interesting directions can be explored and experimented, we may extend this technique on a large real-time dataset in the future.

Academic Contribution. All most every recommendation system is based on anyone's method from popularity, review, rating, etc. nowadays, but in some popular, and large enterprises choose hybrid method due to a huge number of users and budget allocation. It requires a powerful and efficient computing environment which is very costly, about to unaffordable by the small scale of retail sales, Users involvement in all the above methods is very low in ratio as compare to the number of users associated with the system. The proposed system is very useful for small scale enterprise and retail shops, where the number of users is low. Every user is important so we can handle it very efficient manner due to consideration of view events in the generation of the ranking score of any item.

Acknowledgments. The first author express the profound sense of gratitude and deep sentiments to the supervisor Dr. O.P.Rishi, Associate Professor, Department of Computer science and Informatics, University of Kota, Kota, for their generous help, perpetual encouragement and abundant interest towards the paper. It was their benign direction that saw the initiation, conduction, and completion of the paper. The first author also thanks the publisher for the INFO-TeX mailing list for the valuable indirect assistance he received, which is very helpful for writing this paper.

\section{REFERENCES}

[1] Z. Zeng,An Intelligent E-Commerce Recommender System Based on Web Mining, International Journal of Business and Management (IJBM), Vol. 4, No. 7, pp 10-14, July 2009.

[2] Pitkow et AL,PERSONALIZED SEARCH A contextual computing approach may prove a breakthrough in personalized search efficiency, COMMUNICATIONS OF THE ACM Vol. 45, No. 9,2002.

[3] C. C. Aggarwar, Recommender Systems: Text Book, Springer,( 2016) DOI 10.1007/978-3-319-29659-3.13.

[4] M. Balabanovic and Y. Shoham, Content-Based Collaborative Recommendation Comm. ACM, Mar. 1997, pp. 66-72.

[5] S. Panagiotis, A. Nanopoulos, A. N. Papadopoulos, and Y. Manolopoulos, Collaborative recommender systems: Combining effectiveness and efficiency Expert Systems with Applications 34.4 (2008) pp 2995-3013.

[6] H. Sadreazami, A. Asif and A. Mohammadi, Iterative Graph-Based Filtering for Image Abstraction and Stylization, in IEEE Transactions on Circuits and Systems II: Express Briefs, vol. 65, no. 2, pp. 251-255, Feb. 2018.

[7] Y. Dai, H. Ye, And S. Gong, Personalized Recommendation Algorithm Using User Demography Information, 2009 Second International Workshop8on Knowledge Discovery and Data Mining, Moscow, 2009, pp. 100-103.

[8] A.U. Martliong and N.M.S. Iswari, Rancang Bangun System Rekomendasi Restoran Menggunakan Metode AHP dan VIKOR pada platform Line, J.Ultim. Comput. Vol 10 no. 1,(2018), pp 27-33.

[9] Z. Wang, J. Liao, Q. CaO And H. Q, Friendbook A Semantic based friend recommendation for Social Networks., IEEE Transaction, Mob. Comput., vol. 14 no. 3, (2015) pp. 538-551.

[10] S.Chen, S. Owusu AND L. ZHOU , Social Network Based Recommendation Systems: A short Survey, doi:10.1109/SocialCom.2013.134,2013

[11] C.A. Gomez Uribe and N. Hunt, LU-The Netflix Recommender System: Algorithms, Business Value, and Innovation, ACM Trans. Manag. Inf. Syst. 2015

[12] N.M.S. Iswari, Wella AND A. Rusli, Product Recommendation for e-Commerce System Based on Ontology, IEEE Explore, ICORIS -2019

[13] D. Wang, Y. Lianga, D. Xua, Xi. Feng, R. Guan, A content-based recommender system for computer science publications, Knowledge-Based Systems 157 (2018) 1-9.

[14] A. Kanakia, Z. Shen, D. Eide, K. Wang, A Scalable Hybrid Research Paper Recommender System for Microsoft Academic, ACM ISBN 978-1-4503-6674-8/19/05. (2019) https://doi.org/10.1145/3308558.3313700

[15] A. Koundria, O. Nounli, M. Yahya, H. Al-Shamri, A Multi-criteria Collaborative Filtering Recommender System Using Learning-to-Rank and Rank Aggregation, Arabian Journal for Science and Engineering September 2019 https://doi.org/10.1007/s13369-019-04180-3

[16] M. K. Singh, O. P. Rishi, S. Awasthi, A. P. Srivastava and S. Wadhwa, Classification and Comparison of Web Recommendation Systems used in Online Business, 2020 International Conference on Computation, Automation and Knowledge Management (ICCAKM), Dubai, United Arab Emirates, 2020, pp. 471-480.

[17] L. Yan, Personalized Recommendation Method for E-commerce Platform based on Data Mining Technology, Proc.-2017Int. Conf. Smart Grid Electr. Autom ICSGEA 2017, Vol. 2017- Jan. (2017), pp 514-517.

[18] D.Yu, Y. Mu, AND Y. Jin, Rating Prediction using review text with underlying sentiment,Information Processing Letters,117, (2015), pp 10-18.

[19] P.Lops, M.D.Gemmis, and G.Semeraro,, Content-based recommender systems: state of the art and trends, ", in Recommender Systems Handbook, Springer, (2011), pp. 73-105. 
[20] S.S. Li and E. Karahanna, A review and future directions online recommendation system in B2C E-commerce Context.Journal of Association for Information Systems Online Recommendation Systems in B2C E-commerce Context. 16(2),(2015), pp 72-107.

[21] J. McAuley, C. Targett, Javen and A.V.D Hengel, Image-based Recommendations on styles and substitutes, SIGIR-15, August 09-13,(2015), Santiago, Chile.

[22] Y. Zhang, Q. Ai, X. Chen and P. Wang, Machine Learning and Knowledge Discovery in Databases-Part III, Springer (2018)

[23] |ONLINE|,Available: https//pandas.pydata.org, Accessed September 21,2018.

[24] Hong, Bo, Yu, MengChen ,A collaborative filtering algorithm based on correlation coefficient, Neural Computing and Applications, November 2018, DOI 10.1007/s00521-018-3857-7.

[25] A. Gunawardana, G. Shani,Evaluating recommender systems, in Recommender Systems Handbook, Springer, 2015 , pp. $265-308$

[26] S Rendle. C Freudenthaler, Z. Gantner, L. Schmidt-Thieme, BPR: Bayesian personalized ranking from implicit feedback, In Proceedings of the Twenty-Fifth Conference on Uncertainty in Artificial Intelligence, Montreal, QC, Canada, 18-21 June 2009; pp. 452-461.

[27] J.McAuley, J. LeSKOvec, Hidden: factors and hidden topics: understanding rating dimensions with review text, In Proceedings of the 7th ACM Conference on Recommender Systems, Hong Kong, China,12-16 October 2013; pp. 165-172.

[28] R. He, J. McAuley ,VBPR: Visual Bayesian Personalized Ranking from Implicit Feedback, In Proceedings of the 30th AAAI Conference on Artificial Intelligence, Phoenix, AZ, USA, 12-17 February 2016; pp. 144-150.

[29] L. Zheng, V. Noroozi, P.S. Yu, Joint deep modeling of users and items using reviews for recommendation, In Proceedings of the Tenth ACM International Conference on Web Search and Data Mining, Cambridge, UK, 6-10 February 2017; pp. 425-434.

[30] Y. Zhang, Q. AI, X.Chen, W.B. Croft,Joint representation learning for top-n recommendation with heterogeneous information sources, In Proceedings of the 2017 ACM Conference on Information and Knowledge Management, Singapore, 6-10 November 2017; pp. 1449-1458.

Edited by: Anand Nayyar

Received: Mar 10, 2020

Accepted: Jul 6, 2020 